\title{
Mechanisms of reduced left ventricular filling rate in coronary artery disease
}

\author{
W K K HUI, D G GIBSON \\ From the Brompton Hospital, London
}

SUMMARY To identify mechanisms underlying slow left ventricular filling in coronary artery disease, left ventriculograms from 93 patients and 18 normal subjects were digitised frame by frame and global and regional function analysed. In 54 patients peak normalised filling rates were above the lower $95 \%$ confidence limit of normal $\left(2.9 \mathrm{~s}^{-1}\right)$ and in 39 they were below. Patients with slow filling had a lower ejection fraction, a higher end systolic volume, and less overall shape index change, although a larger percentage occurred during isovolumic relaxation owing to asynchronous relaxation. Stroke volume was not significantly different. Slow outward wall motion was associated with increased cavity volume and systolic hypokinesis. Wall motion was also appreciably asynchronous, with wide spreads in the times of peak outward velocity and termination of rapid outward movement between regions. Early outward movement usually started in the anterior region, with peak velocity occurring before mitral valve opening, and significantly earlier than that in the apex or the inferior region. Ventricular oscillations occurred during filling in 23 patients. This asynchronous wall movement was unrelated to the distribution of coronary artery disease, systolic hypokinesis, or $Q$ waves on electrocardiograms but was similar to that occurring in mitral stenosis. The main causes of slow left ventricular filling in patients with coronary artery disease appear to be $(a)$ failure to achieve a normal low end systolic volume, with associated loss of physiological changes in left ventricular cavity shape, and $(b)$ incoordinate wall motion during isovolumic relaxation which dissipates energy normally coupled to rapid ventricular filling. The resulting slow and asynchronous wall motion may have clinical implications especially when the time available for left ventricular filling is short.

A reduced rate of left ventricular filling is one of several diastolic abnormalities seen in patients with ischaemic heart disease. It was shown with cineangiography by Hammermeister and Warbasse ${ }^{1}$ and has been confirmed by radionuclide angiography. ${ }^{23}$ Although its cause is not clear, it may be clinically important in limiting exercise tolerance in patients with coronary artery disease. We have thus investigated its mechanisms by using cineangiography to analyse left ventricular diastolic wall motion in detail.

\section{Patients and methods}

One hundred and eleven patients who underwent cardiac catheterisation at this hospital for investigation of chest pain were studied. In 88 patients coronary artery disease was confirmed by coronary arterio-

WKKH was in receipt of a Croucher Foundation fellowship

Accepted for publication 5 July 1983 graphy. Haemodynamics and coronary arteriograms were normal in 18 patients, and these were taken as controls. A further five patients with confirmed previous myocardial infarction and suspected left ventricular aneurysm who underwent left ventriculography only were included in the coronary artery disease group, making a total of 93 patients.

There were 12 men and six women in the control group (age 29-69 years). The coronary artery disease group consisted of 82 men and 11 women (age range 36-69 years); all were in sinus rhythm. Five patients in the control group (normal subjects) and 43 patients in the coronary artery disease group were taking a beta blocking drug at a minimum equivalent dose of $80 \mathrm{mg} /$ day propranolol when angiography was performed. The frequency and location of pronounced $Q$ waves (defined as those with negative deflection of at least $2 \mathrm{~mm}$ and duration of at least $40 \mathrm{~ms}$ ) on electrocardiograms and the pattern of coronary artery involvement were noted. 
Left ventricular angiograms were performed before coronary arteriography in either the $30^{\circ} \mathrm{RAO}$ or anteroposterior projection with the patient supine. A bolus of $20-40 \mathrm{ml}$ of Urografin 400 was injected at a rate of $15-20 \mathrm{ml} / \mathrm{s}$ through a retrograde catheter into the left ventricle with cine film exposed at 50 frames/s. Calibration was performed using a grid at midchest level. No patient had severe mitral regurgitation. Only sinus beats were selected for analysis. Ectopic and postectopic beats were not studied.

Coronary angiography was carried out using standard techniques. Multiple views were taken of the coronary arteries, with at least four projections for the left and two projections for the right coronary artery.

\section{ANALYSIS OF ANGIOGRAMS}

Left ventricular angiograms were digitised using a Prime 750 computer and a Summagraphics digitiser. ${ }^{4}$ The information was stored on disc for further calculations. Mitral valve opening was taken as the time of first appearance of unopacified blood in the left ventricle. Cavity area was calculated by numerical integration and plotted throughout the cardiac cycle. Volume estimates were derived from area ${ }^{5}$ and were used to calculate ejection fractions and filling rates. A three point moving average filter was applied. Left ventricular shape index 6 was calculated and overall changes for the whole cardiac cycle, as well as those during the isovolumic relaxation period-the interval between the time of minimum cavity area and mitral valve opening were noted.

For the purpose of regional wall motion analysis the end diastolic left ventricular outline was divided into 40 equally spaced points starting from the border of the aortic root adjacent to the mitral valve, and proceeding in an anticlockwise direction. From each, the nearest point on the end systolic frame was sought, and along the line thus defined endocardial position in each cine frame was measured and expressed in $\mathrm{mm}$ from its end diastolic position. The resulting 40 plots of wall movement against time were displayed either stacked obliquely, as an isometric display with superimposed isochrones to represent the timing of successive cine frames, or as a contour map, in which each contour represents inward movement of the endocardium by $1 \mathrm{~mm}$ from its end diastolic position. On both displays the times of minimum cavity area and mitral valve opening were marked. The left ventricular endocardial outline was divided into three regions, inferior, apical, and anterior, corresponding to segments $1-14,15-24$, and $25-40$ respectively from the 40 points on the diastolic frame. Separate plots of wall motion and its rate of change were obtained from the midpoint of the three regions and the following measurements made for each:

(1) Systolic function was measured as the overall amplitude of inward movement in the three regions during the interval between end diastole and minimum cavity area. It was classified as normal or reduced if it was below the lower $95 \%$ confidence limit of normal for the region in question.

(2) Peak velocity of outward wall movement and timing of its occurrence after mitral valve opening. If peak velocity occurred before mitral valve opening the time was expressed as a negative value. The time interval from the earliest to the latest occurrence of peak velocity in the three regions, the spread, was also determined.

(3) Rapid outward wall movement was taken as having ended when its velocity had dropped to less than $3 \mathrm{~cm} / \mathrm{s}$. The time when this occurred for each region and its spread between regions were noted.

(4) Segmental wall movement was assessed from isometric and contour plots. ${ }^{78}$ The following specific abnormalities during diastole were sought: (a) inward movement of more than $2 \mathrm{~mm}$ or outward movement of more than $6 \mathrm{~mm}$ involving four or more consecutive segments during isovolumic relaxation; movement of this sort represents an abnormal change in cavity shape during isovolumic relaxation; (b) inward wall movement of more then $2 \mathrm{~mm}$ after mitral valve opening - that is, during the period of ventricular filling ${ }^{8}$; this was taken as evidence of an oscillation of the ventricular cavity.

Comparisons were made between the three different regions of the ventricle and also between controls and patients with coronary artery disease.

\section{STATISTICAL METHOD}

Results were analysed using Student's $t$ test, the Mann-Whitney U test, $\chi^{2}$ test, or Fisher's exact probability test, as appropriate.

\section{Results}

\section{CONTROLS}

\section{Overall function (Table 1)}

The mean values for heart rate, ejection fraction, peak diastolic filling rate, end diastolic volume, peak filling rate normalised to unit end diastolic volume, time of peak filling, overall shape index change, shape index increase during filling, and the percentage of overall shape index change during isovolumic relaxation were all within previously published normal limits. ${ }^{89}$ Correlation between stroke volume and peak filling rate was close $(r=0.94)$, although this was not present when normalised filling rate was considered.

\section{Regional function (Table 2)}

Peak velocities of outward movement for the three regions were again similar to previously published values, and there was no significant difference be- 
Table 1 Overall function in normal subjects and patients with coronary artery disease. Values are means $\pm S D$

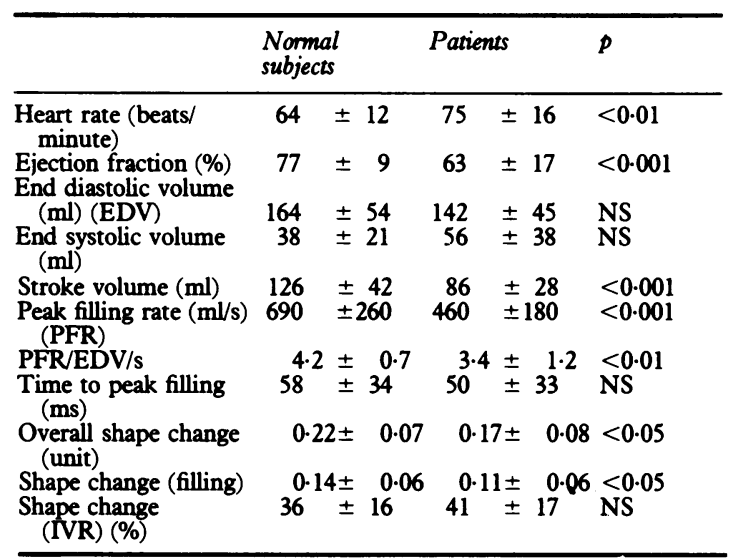

PFR/EDV, peak filling rate normalised to unit end diastolic volume; IVR, isovolumic relaxation; NS, not significant.

tween the values for the three regions. Outward wall motion was synchronous. It usually started before, but peak velocities were always achieved after, mitral valve opening with the exception of the inferior region in one control. This uniformity was reflected by the narrow spread of around $40 \mathrm{~ms}$ both in the timing of occurrence of peak outward velocity and termination of rapid outward movement in the three regions. No abnormal segmental wall movement was detected in any of the controls.

\section{Influence of beta blockade}

No significant difference could be shown between the five controls who were taking a beta blocking drug, and the remaining 13 with respect to any measurement made in this study.

Table 2 Regional function in normal subjects and patients with coronary artery disease. Values are means $\pm S D$

\begin{tabular}{lccl}
\hline & $\begin{array}{c}\text { Normal } \\
\text { subjects }\end{array}$ & Patients & $p$ \\
\hline Peak velocity of outward wall movement $(\mathrm{cm} / \mathrm{s})$ & \\
Anterior & $11 \pm 4$ & $10 \pm 4$ & NS \\
Apical & $11 \pm 5$ & $8 \pm 5$ & $<0.02$ \\
Inferior & $13 \pm 4$ & $8 \pm 4$ & $<0.001$ \\
Time of peak outward velocity from & MVO(ms) & \\
Anterior & $39 \pm 33$ & $-6 \pm 66$ & $<0.01$ \\
Apical & $40 \pm 27$ & $29 \pm 67$ & NS \\
Inferior & $43 \pm 27$ & $36 \pm 55$ & NS \\
Spread & $43 \pm 25$ & $105 \pm 65$ & $<0.001$ \\
Time of termination & of rapid & outward wall & movement from \\
MVO(ms) & & & \\
Anterior & $101 \pm 28$ & $49 \pm 76$ & $<0.01$ \\
Apical & $94 \pm 27$ & $70 \pm 71$ & NS \\
Inferior & $89 \pm 38$ & $81 \pm 63$ & NS \\
Spread & $43 \pm 21$ & $106 \pm 67$ & $<0.001$ \\
\hline
\end{tabular}

MVO, mitral valve opening; NS, not significant.

\section{CORONARY ARTERY DISEASE}

Clinical details

Coronary arteriography showed single vessel disease in 12 patients, double vessel disease in 27 , and triple vessel disease in 49 . Of the affected vessels, 59 were occluded; severe stenoses were present in 91 , and a $50-70 \%$ stenosis in a further 44 . Sixty-eight vessels had a stenosis of $50 \%$ or were normal. $Q$ waves were absent in 45 patients and were present in 46; of the latter, 13 were anterior, 23 inferior, and three lateral, and in seven both anterior and inferior leads were involved. Reduced amplitude of systolic wall motion was present in the anterior region in 11 patients, the apex in 35 , and the inferior region in 35.

\section{Overall function (Table 1)}

The peak diastolic filling rate was significantly lower than normal whether expressed in absolute terms or normalised to unit end diastolic volume. The time of peak filling from mitral valve opening was not, however, different from normal. Overall shape index change during the cardiac cycle was less than normal, as was the shape index increase during filling. As has previously been reported, there was close correlation between ejection fraction and end systolic volume $(r=0.91)$; that between peak filling rate and stroke volume $(0.63)$ was significantly less than in the controls. Correlation was absent between stroke volume and normalised filling rate $(r=0 \cdot 11)$. Although heart rate was significantly higher than in normal subjects, the only variable measured in the present study with which it correlated was one whose values did not differ significantly between controls and patients with ischaemic heart disease, the time to peak filling, where the correlation coefficient was -0.31 . In the remainder of the variables measured there was no significant correlation with heart rate (correlation coefficients between -0.18 and +0.095 ).

\section{Regional function (Table 2)}

Peak velocity of outward movement of the anterior region was normal while that of the apex and inferior region were reduced. When the three regions themselves were compared, the velocity in the anterior region was again greater $(p<0.002 v$ apical and $p<0.001$ $v$ inferior region). Not surprisingly, the peak velocity of outward wall motion was reduced in regions showing systolic hypokinesis. For the anterior region, the mean (SD) velocities were $11 \pm 4 \mathrm{~cm} / \mathrm{s}$ for those regions with normal systolic function as against $5 \pm 2$ for those in which it was depressed $(p<0.001)$. For the apex, the corresponding figures were $10 \pm 5$ and $5 \pm 3$ $\mathrm{cm} / \mathrm{s}(\mathrm{p}<0.001)$, and for the inferior wall $9 \pm 4$ and $6 \pm 3 \mathrm{~cm} / \mathrm{s}(p<0.001)$. When the amplitude of systolic wall motion was maintained, therefore, regional outward velocities did not differ significantly from nor- 
mal in patients with coronary artery disease in the anterior region and at the apex, but they were still significantly reduced inferiorly $(p<0.01)$. The times of occurrence of peak outward velocity and termination of rapid outward movement in the three regions were not only different from normal but also from region to region. Outward movement in the anterior region frequently preceded that in the other two regions, and in 32 patients this was reached before mitral valve opening occurred. The differences in timing of occurrence of peak outward velocity between the anterior and the other two regions were statistically significant ( $p<0.001$ for both). These regional differences tended to persist throughout diastole so that termination of rapid outward movement also occurred earlier in the anterior region, although for this event a significant difference was shown only between the anterior and inferior regions $(p<0.01)$.

Further manifestations of asynchronous diastolic wall motion included a much greater spread in the timing of the occurrence of peak outward velocity and termination of rapid outward movement in comparison with normal ( $<<0.001$ for both). None of these aspects of regional asynchrony was affected by the presence of systolic hypokinesis. Abnormal wall motion during isovolumic relaxation was present in 18 patients (Table 3, Fig. 1). Its pattern was similar to that described elsewhere. ${ }^{7}$

\section{Ventricular oscillations (Table 3)}

Abnormal inward wall movement during filling by $2 \mathrm{~mm}$ or more was commonly observed (Fig. 2). It was usually accompanied by compensatory outward movement in another region giving rise to an oscillation involving the ventricle. This occurred in 23 patients, with two patients having oscillations in two regions. In patients with oscillations the timing of peak outward velocity in the segment moving inward initially was delayed to $63 \pm 38 \mathrm{~ms}$ with respect to mitral valve opening against $46 \pm 30 \mathrm{~ms}$ for those without $(p<0.05)$. In all of these patients at least one region reached a peak outward velocity of $8 \mathrm{~cm} / \mathrm{s}$ or more. The spread of both peak and termination of rapid outward movement was significantly greater in those with oscillations (mean (SD) $128 \pm 57 \mathrm{~ms}$ and $134 \pm 65 \mathrm{~ms}$ ) than in those without (mean (SD) $98 \pm 65$ $\mathrm{ms}$ and $97 \pm 66 \mathrm{~ms}, \mathrm{p}<0.05$ for both).

\section{Stratification by filling rate (Tables 4 and 5)}

To identify factors associated with slow filling patients with normalised filling rates above and below the lower $95 \%$ confidence limit of normal were studied separately.

Normal filling rate was present in 54 patients. Absolute filling rate was slightly reduced, but shape index changes in these patients were almost identical
Table 3 Abnormal diastolic wall motions in patients with coronary artery disease. Results are number of patients

\begin{tabular}{lccc}
\hline & \multicolumn{2}{l}{$\begin{array}{l}\text { Isovolumic } \\
\text { relaxation }\end{array}$} & Oscillation \\
\cline { 2 - 3 } & In & Out & \\
\hline Anterior & 1 & 16 & 11 \\
Apical & 9 & 3 & 8 \\
Inferior & 2 & 2 & 6 \\
Total & $11^{\star}$ & $18 \mathrm{t}$ & $23 \ddagger$ \\
\hline
\end{tabular}

*Involvement of both apical and inferior regions in one patient. IInvolvement of both anterior and apical regions in two patients. fInvolvement of both anterior and inferior regions in two patients.

with normal, and the peak velocity of outward motion reduced only in the inferior region. Nevertheless, the greater velocity of outward motion of the anterior wall with respect to the other two persisted. As in the group as a whole, peak rates of outward wall motion were significantly reduced in regions showing systolic hypokinesis $(p<0.001)$. Incoordination in the timing of the peak (spread $89 \pm 59 \mathrm{~ms}$ ) and the end (spread $87 \pm 62 \mathrm{~ms}$ ) of rapid outward movement in the three regions was present, however, despite filling rate being normal. Stroke volume in these patients was $88 \pm 30 \mathrm{ml}$, which was less than normal $(p<0.001)$, though end systolic volume $(40+40 \mathrm{ml})$ was not significantly different.

Peak normalised filling rate was reduced in 39 patients. These values differed significantly from those in patients whose filling rate was normal in that ejection fraction was lower, end diastolic volume higher, and peak filling occurred earlier. End systolic volume was about twice that of normal, so that it was significantly greater than that in patients with coronary artery disease who had normal filling rates, while stroke volume was not significantly different. Although overall shape index changes were less, the proportion of overall change during isovolumic relaxation was increased. Peak outward velocity was decreased in all three regions, particularly when systolic hypokinesis was present, and peak velocity was reached even earlier in the anterior region. The time spread for the occurrence of peak and end of rapid outward wall motion were both more than $120 \mathrm{~ms}$, indicating that the degree of incoordination was much greater than in the patients in whom the filling rate was normal. These time relations were unaffected by the presence of systolic hypokinesis.

\section{Other factors}

Distribution of coronary artery disease-No relation could be found between the distribution of coronary artery disease and filling rate nor indeed any aspect of diastolic wall motion. In particular, the characteristic pattern of early movement of the anterior wall was 

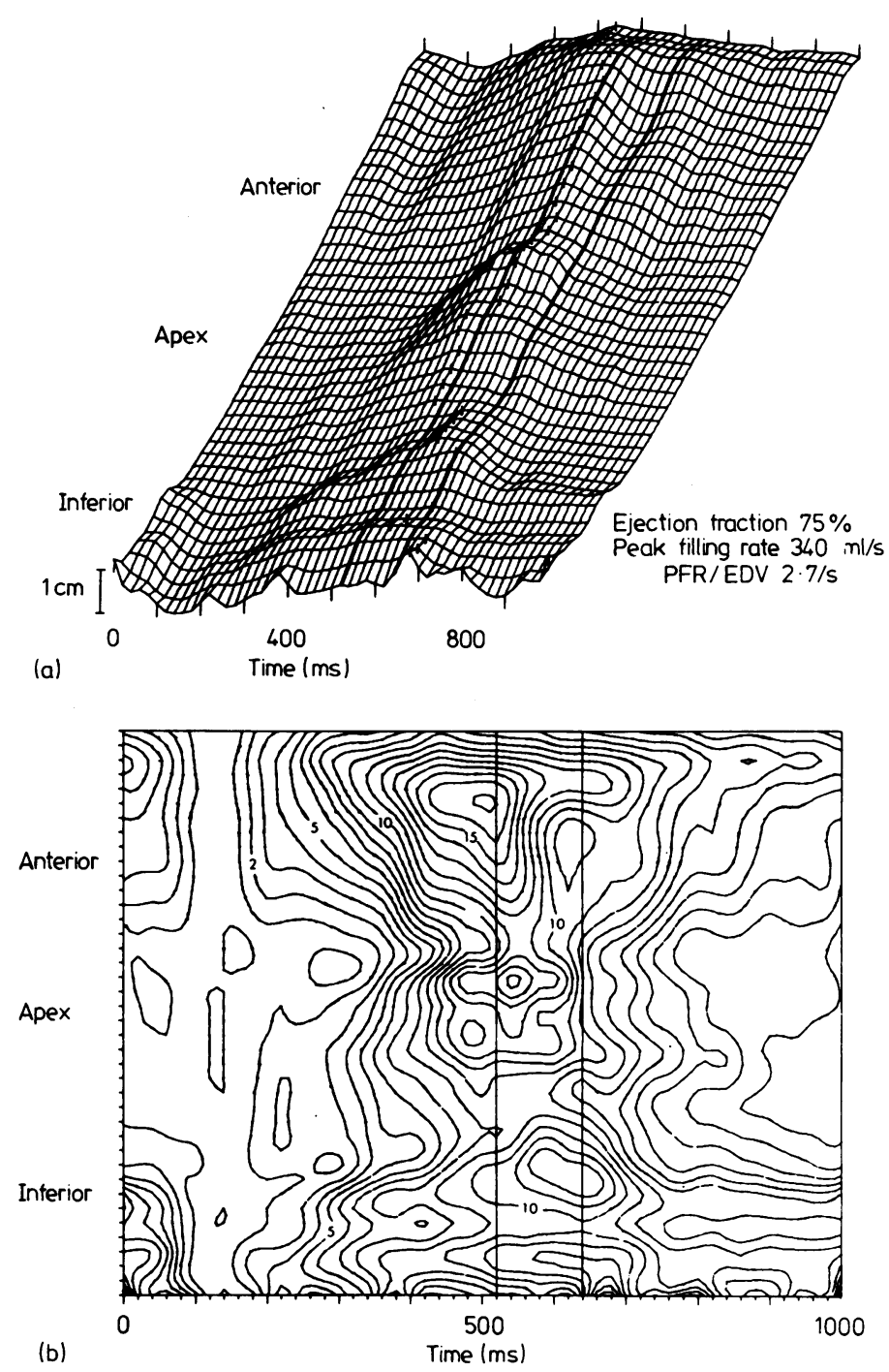

Fig. 1(a) Isometric display of left ventricular endocardial movement with time in a patient with coronary artery disease. The diagonal lines are isochrones corresponding to the times of successive cine frames. The times of minimum cavity area and mitral valve opening are indicated by the two accentuated isochrones. Abnormal early outward movement is evident in the anterior region of the endocardial outline. (b) Contour display from the same data as in (a). Inward wall movement is indicated by contour lines, each representing movement of $1 \mathrm{~mm}$. The distance from the position of the wall in the end diastolic frame is indicated by the numbers. The two vertical lines represent the times of minimum cavity area and mitral valve opening. The abnormal early outward movement in the anterior region is illustrated. unaffected: There was no difference in the pattern of coronary artery disease between the 32 patients whose anterior region reached peak outward velocity before mitral valve opening and the 34 in whom anterior peak outward velocity occurred not only after mitral valve opening but also later than either or both of the other two regions.

Association with $Q$ waves-No association between diastolic wall motion and the presence or distribution of $Q$ waves was found.

Effect of beta blockade-Comparison of results in patients taking and not taking beta blocking drugs showed a slightly higher ejection fraction in those taking a beta blocking drug. This was not considered to be a therapeutic effect but to reflect prescribing habits, in that such drugs were used more sparingly in those patients whose left ventricular function was significantly depressed. Associated with a lower ejection fraction in the untreated patients were a higher end diastolic volume, lower velocities of outward wall motion, and reduced shape index changes. When patients with low ejection fractions were excluded from the untreated group, however, those remaining were found to be no different from the treated group no matter whether overall or regional function was compared. Thus, as in the case of the controls, beta blockade seemed to have no effect on overall filling or regional wall motion. 


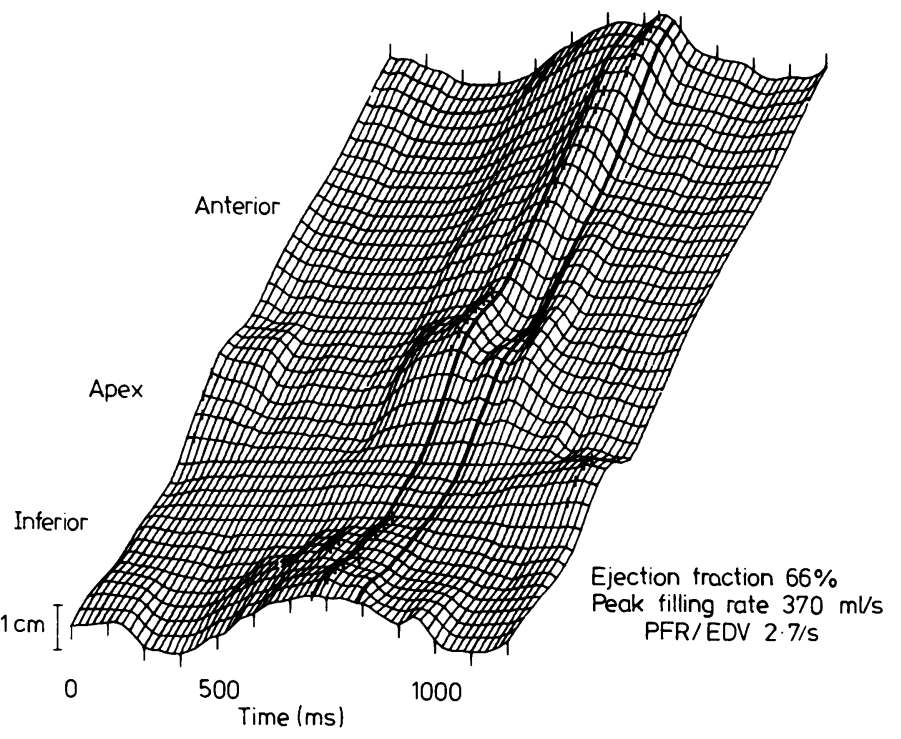

Fig. 2(a) Isometric display of left ventricular endocardial movement from a patient with coronary artery disease. Abnormal early outward movement in the apical region is followed by inward movement after mitral valve opening, resulting in an oscillatory motion. Compensatory movement is seen in the anterior

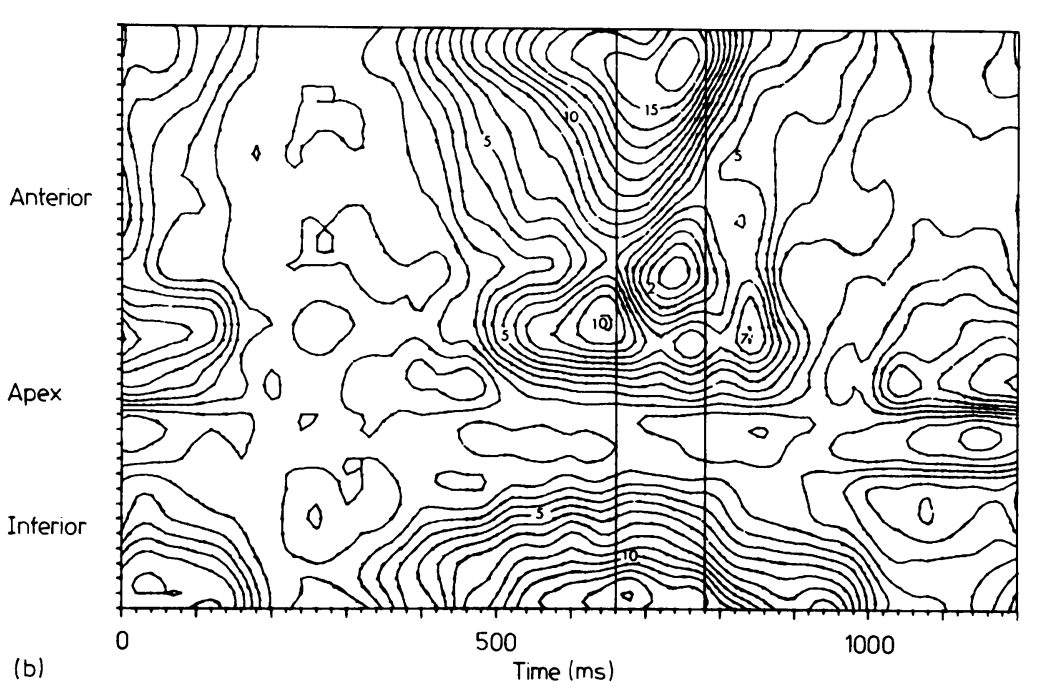
region. (b) Contour display from the same data as in (a). The oscillation at the apex is clearly shown. Note also that after mitral valve opening the normal pattern of wall movement is nowhere to be found in the entire ventricle.

\section{Discussion}

Contrast angiography has advantages for studying left ventricular wall motion during filling in patients with coronary artery disease. Its lateral resolution is greatly superior to that of cross sectional echocardiography or nuclear angiography, and unlike the latter method it is possible to time the onset of mitral valve opening, so that events during isovolumic relaxation can be distinguished from those during filling. Values of peak left ventricular filling rate reported here are slightly higher than those of Hammermeister and Warbasse. ${ }^{1}$ The difference is probably attributable to the method of smoothing used. We used a three point moving average filter, while Hammermeister and Warbasse used a second order nine point polynomial fit. Implementation of this latter technique to our results led to values of peak filling rate about $15 \%$ lower, making agreement between the two studies almost complete.

The normal pattern of left ventricular filling was confirmed in the present study. The ventricular volume curve showed the characteristic division into the early period of rapid filling, a mid-diastolic period of diastasis, and a further increase during left atrial systole. ${ }^{10}$ Peak rates of volume increase were always 
Table 4 Overall function in patients with coronary artery disease subgrouped according to filling rate. Vales are means $\pm S D$

\begin{tabular}{|c|c|c|c|c|c|}
\hline \multirow[b]{3}{*}{$\begin{array}{l}\text { Heart rate (beats/minute) } \\
\text { Ejection fraction (\%) } \\
\text { End diastolic volume (ml) } \\
\text { End systolic volume (ml) } \\
\text { Stroke volume (ml) } \\
\text { Peak filling rate (ml/s) } \\
\text { PFR/EDV/s } \\
\text { Time to peak filling (ms) } \\
\text { Overall shape change } \\
\text { Shape change (filling) } \\
\text { Shape change (IVR) }(\%)\end{array}$} & \multirow{2}{*}{$\begin{array}{l}\text { Normal } \\
\text { subjects }\end{array}$} & \multirow[t]{2}{*}{$p$} & \multicolumn{3}{|l|}{ Patients } \\
\hline & & & $\begin{array}{l}\text { Normal } \\
P F R / E D V\end{array}$ & $p$ & $\begin{array}{l}\text { Low } \\
P F R / E D V\end{array}$ \\
\hline & $\begin{array}{c}64 \pm 12 \\
77 \pm 9 \\
164 \quad \pm 54 \\
38 \pm 21 \\
126 \pm 42 \\
690 \pm 260 \\
4 \cdot 2 \pm 0.7 \\
58 \pm 34 \\
0.22 \pm 0.07 \\
0 \cdot 14 \pm 0.06 \\
36 \quad \pm 16\end{array}$ & $\begin{array}{l}<0.01 \\
<0.05 \\
<0.01 \\
\text { NS } \\
<0.001 \\
<0.05 \\
\text { NS } \\
\text { NS } \\
\text { NS } \\
\text { NS } \\
\text { NS }\end{array}$ & $\begin{array}{c}76 \pm 16 \\
70 \pm 13 \\
128 \pm 42 \\
40 \pm 40 \\
88 \pm 30 \\
530 \pm 190 \\
4 \cdot 2 \pm 0 \cdot 9 \\
58 \pm 32 \\
0 \cdot 20 \pm 0.07 \\
0.13 \pm 0.06 \\
37 \pm 15\end{array}$ & $\begin{array}{l}\text { NS } \\
<0.001 \\
<0.001 \\
<0.001 \\
\mathrm{NS} \\
<0.001 \\
<0.001 \\
<0.01 \\
<0.001 \\
<0.001 \\
<0.01\end{array}$ & $\begin{array}{c}74 \pm 16 \\
53 \pm 16 \\
162 \pm 41 \\
78 \pm 26 \\
83 \pm 26 \\
360 \pm 100 \\
2.3 \pm 0.5 \\
39 \pm 31 \\
0.13 \pm 0.06 \\
0.08 \pm 0.05 \\
47 \pm 18\end{array}$ \\
\hline
\end{tabular}

Abbreviations as in Table 1 .

recorded during early diastole. The normal pattern of wall motion during this period is synchronous, with no more than $40 \mathrm{~ms}$ between the timing of the peak or the end of outward motion between different regions of the cavity. The whole period of rapid outward motion ends within $100 \mathrm{~ms}$ of mitral valve opening. This value is probably of no great physiological importance at rest, but during exercise it corresponds almost exactly with the $100-120 \mathrm{~ms}$ available for filling as measured from the duration of mitral valve opening in healthy subjects. ${ }^{11}$

The commonest abnormality of filling in patients with coronary artery disease was loss of the normal synchronous pattern of wall motion. This could be quantified as an increase in the spread of the timing of occurrence both of peak outward motion and the end of rapid filling between the three regions of the cavity. Both time intervals were increased from about $40 \mathrm{~ms}$ in controls to a mean of $105 \mathrm{~ms}$ in patients with coronary artery disease. When identified in this way, values outside the $95 \%$ confidence limits of normal were seen in $52(56 \%)$ patients. In addition, this distur- bance to wall motion was not random, but the anterior wall moved outward in advance of the apical and inferior regions. This pattern was consistently seen and was unaffected by beta blockade, the distribution of coronary artery lesions, or $Q$ waves on the electrocardiogram. Incoordinate wall motion during filling was present even in patients whose ejection fraction was greater than $60 \%$ or whose peak filling rate was identical with normal. Incoordinate wall motion during ventricular filling is thus a common disturbance in patients with coronary artery disease.

A second and rather less common finding was a reduction in the peak rate of ventricular filling with values below the lower $95 \%$ confidence limit of nor$\mathrm{mal}$ in $40 \%$ of the patients. This value is similar to that reported by Reduto et al., ${ }^{2}$ using first pass nuclear angiography, but less than that of Bonow et al. ${ }^{3}$ based on gated scans. To study the associations of slow ventricular filling in more detail, patients with values below the $95 \%$ confidence limit were compared with those in whom the filling rate was normal. A slow filling rate was associated with a reduced ejection frac-

Table 5 Regional function in patients with coronary artery disease subgrouped according to filling rate. Values are means $\pm S D$

\begin{tabular}{|c|c|c|c|c|c|}
\hline & \multirow{2}{*}{$\begin{array}{l}\text { Normal } \\
\text { subjects }\end{array}$} & \multirow[t]{2}{*}{$p$} & \multicolumn{3}{|l|}{ Patients } \\
\hline & & & $\begin{array}{l}\text { Normal } \\
P F R / E D V\end{array}$ & $p$ & Low \\
\hline \multicolumn{6}{|c|}{ Peak velocity of outward wall movement $(\mathrm{cm} / \mathrm{s})$} \\
\hline Anterior & $11 \pm 4$ & NS & $12 \pm 4$ & $<0.002$ & \\
\hline Apical & $11 \pm 5$ & NS & $10 \pm 5$ & $<0.001$ & $6 \pm 4$ \\
\hline Inferior & $13 \pm 4$ & $<0.001$ & $9 \pm 4$ & $<0.002$ & $6 \pm 3$ \\
\hline \multicolumn{6}{|c|}{ Time of peak outward velocity from $\mathrm{MVO}(\mathrm{ms})$} \\
\hline Anterior & $39 \pm 33$ & NS & $11 \pm 63$ & $<0.01$ & $-30 \pm 64$ \\
\hline Apical & $40 \pm 27$ & NS & $31 \pm 65$ & NS & $25 \pm 70$ \\
\hline Inferior & $43 \pm 27$ & NS & $45 \pm 46$ & NS & $26 \pm 64$ \\
\hline Spread & $43 \pm 25$ & $<0.002$ & $89 \pm 59$ & $<0.01$ & $127 \pm 67$ \\
\hline \multicolumn{6}{|c|}{ Time of termination of rapid outward wall movement from $M V O(\mathrm{~ms})$} \\
\hline Anterior & $101 \pm 28$ & & $70 \pm 68$ & $<0.002$ & $20 \pm 79$ \\
\hline Apical & $94 \pm 27$ & NS & $79 \pm 65$ & NS & $57 \pm 77$ \\
\hline Inferior & $89 \pm 38$ & NS & $93 \pm 48$ & NS & $63 \pm 76$ \\
\hline Spread & $43 \pm 21$ & $<0.01$ & $87 \pm 62$ & $<0.002$ & $132 \pm 66$ \\
\hline
\end{tabular}

MVO, mitral valve opening; NS, not significant. 

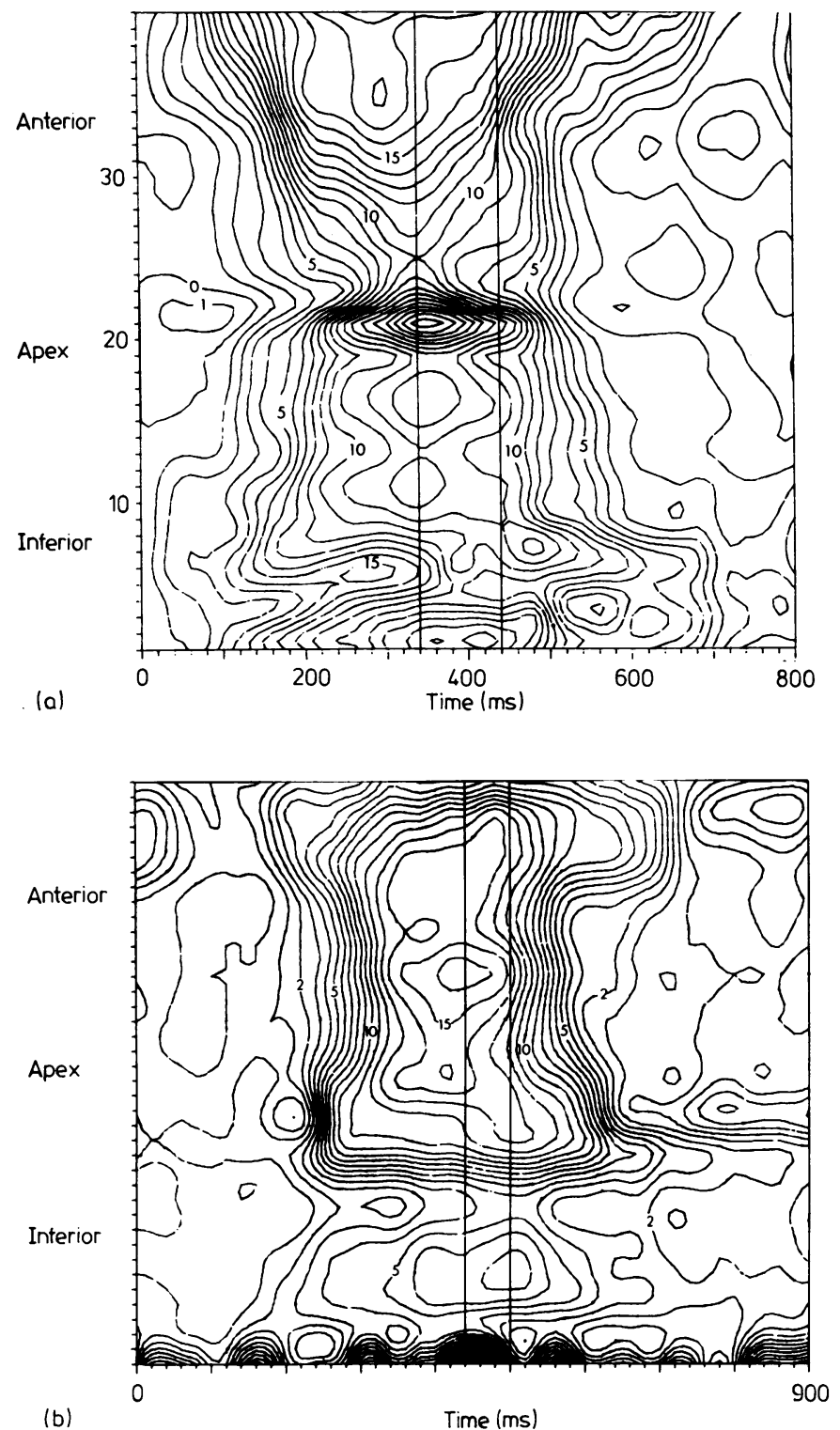

Fig. 3 Contour displays (a) from a normal subject (note the rapid synchronous outward wall movement after mitral valve opening); and $(b)$ from a patient with an inferior scar (note the absence of a change in cavity shape and normal filling pattern in apical and anterior regions despite inferior hypokinesis). tion, raising the possibility that it was simply the consequence of a low stroke volume, particularly since stroke volume and filling rate were strongly correlated in healthy subjects. Our results give no support to this suggestion. Stroke volume was equally depressed in both groups of patients with coronary artery disease, whether or not their filling rate was normal. The component of ejection fraction most closely related to filling rate was end systolic volume, which was normal in patients who had normal filling rates but was doubled in those with slow filling rates. A second possible mechanism of a reduced filling rate is that it was the direct effect of slow outward wall motion due to some regional abnormality of relaxation or compliance. ${ }^{23}$ Our results exclude the need for this hypothesis. Depressed values of peak outward wall motion could be explained in part by their association with an increased end systolic volume. Simple geometrical considerations dictate that at a constant filling rate, the peak rate of outward wall motion is inversely proportional to the two thirds power of cavity volume. A second factor predisposing to slow outward wall 
motion was a reduced amplitude of inward motion in the same segment during systole, independent of any depression of ejection fraction. Slow outward movement of endocardium is thus to be regarded as a consequence of increased end systolic volume and local systolic disease and not to be considered as a primary cause of slow filling. Another possible mechanism, incoordinate wall motion, was a common finding and was present to a greater degree in the patients who had slow filling rates. A surprising feature, however, was its remarkably uniform nature from case to case, regardless of the sites of coronary artery lesions or distribution of $Q$ waves on the electrocardiogram. The pattern of wall motion was almost identical to that seen in a group of patients with mitral stenosis, ${ }^{8}$ in whom the left ventricular filling rate was reduced to a similar extent. It is generally agreed that in mitral stenosis the main disturbance to filling is the mitral stenosis itself, 12 so that here asynchronous wall motion is the result and not the cause of slow filling. In patients with coronary artery disease the pattern was the same, with anterior wall preceding the apex and inferior walls, so that although local ischaemic damage cannot be excluded it appears that in our patients also incoordinate wall motion should be regarded as a secondary phenomenon.

Another major difference between those patients with a normal and reduced filling rate became apparent when cavity shape was considered. Normally cavity shape in a single projection becomes less circular during systole, and this is reflected in a reduction in shape index. ${ }^{6}$ The extent of this change was normal in the patients with a normal filling rate, but was reduced when filling rate was low and end systolic volume abnormally increased. In patients with a normal filling rate the volume increase during rapid filling is accommodated in part by a change in cavity shape to a more spherical configuration, so that the requirement for myocardial distension is correspondingly reduced. Loss of this mechanism, which is closely related to a reduction in ejection fraction, ${ }^{6}$ is thus likely to interfere appreciably with filling. Not only was the overall extent of changes in cavity shape during the cardiac cycle reduced in patients who had slow filling rates but the proportion occurring during isovolumic relaxation was increased. Changes in cavity shape during isovolumic relaxation are common in patients with coronary artery disease and are independent of hypokinesis during ejection. They are due to asynchronous relaxation causing premature outward movement of endocardium before mitral valve opening. ${ }^{713}$ The effect of these disturbances on filling can be assessed from a comparison of Fig. 2(b) with Fig. 3(b), both from patients with right coronary artery disease and inferior hypokinesis during systole, with reference to the filling pattern in a normal sub- ject shown in Fig. 3(a). In Fig. 2(b), a change in cavity shape during isovolumic relaxation caused almost complete disruption of the pattern of filling around the entire cavity outline, whereas in Fig. 3(b), in which there is no isovolumic shape change, wall motion, though absent inferiorly, is virtually normal elsewhere. Finally, as in mitral stenosis, slow filling is associated with oscillations of the ventricular wall which are characterised by simultaneous inward and outward movement of endocardium in different regions of the cavity, at a frequency of $3-4 \mathrm{~Hz}$. This particular type of wall motion abnormality has received little attention in published reports, although its presence has previously been invoked to explain changes in diastolic events with drugs or other manoeuvres. ${ }^{14} \mathrm{~A}$ series of diastolic disturbances can thus be shown in patients with coronary artery disease. The dominant factor predisposing to slow filling appeared to be failure to achieve the normal low end systolic volume, so that the physiological changes in cavity shape underlying normal filling did not occur. Superimposed are the effects of asynchronous relaxation. These further impair diastolic function by allowing outward motion of endocardium before mitral valve opening and so dissipate restoring forces normally coupled to rapid filling. Reduced stroke volume or ejection fraction do not appear to be primary determinants, whereas slow and incoordinate outward wall motion during filling and ventricular oscillations are likely to be secondary effects of an increased cavity size and reduced filling rate.

With this information, it is possible to assess the position of slow filling within the whole series of disturbances of diastolic function seen in patients with coronary artery disease. Reduction in ejection fraction in patients with coronary artery disease is strongly correlated with loss of normal shape index changes ${ }^{6}$ and thus with impaired filling. Shape changes may be compromised, 6 however, and disturbances of isovolumic relaxation may appear ${ }^{7}$ when ejection fraction is still maintained, suggesting that abnormalities of diastolic function may be more sensitive indicators of ventricular disease. Abnormal ventricular compliance has frequently been reported in patients with coronary artery disease, ${ }^{15-17}$ but is is unlikely to be the cause of slow filling. Peak filling occurs within $100 \mathrm{~ms}$ of mitral valve opening, when ventricular pressure is still falling, whereas the simple elastic behaviour implicit in the term compliance is evident only late in diastole. Correlation between impaired compliance would not, however, be unexpected since if shape changes are reduced the extent of myocardial distension and thus the increase in diastolic pressure required to accommodate a given stroke volume will be increased. Superimposed on these effects are those of incoordinate wall motion, whose pattern was inde- 
pendent of any direct manifestations of the coronary artery disease itself. Normal outward wall motion during filling is largely determined by rapid myocardial thinning. Rapid thinning can be dissociated from filling during incoordinate relaxation in patients with coronary artery disease ${ }^{13}$ and so is likely to be energised by elastic forces generated during the previous systole. Its extent varies with position in the cavity, ${ }^{18}$ being maximum in the mid-portion of the free wall. We suggest that these normal regional differences in function are the basis of the incoordinate wall motion seen during filling in patients with coronary artery disease, since a similar sequence of the anterior wall preceding the apex and inferior wall is seen in mitral stenosis ${ }^{8}$ and in non-rheumatic mitral regurgitation. ${ }^{19}$ The incoordinate filling in patients with ischaemic heart disease is thus not the direct effect of regional ischaemia, necrosis, or scarring but results from unmasking of the usually unobtrusive mechanisms responsible for synchronous filling in normal subjects. From their multifactorial origin it seems likely that the extent of these diastolic disturbances will vary from case to case, and their response to pharmacological intervention will be variable. Since they are not related to acute ischaemia they may be expected to persist after successful bypass grafting, when their presence may contribute appreciably to the limitation of exercise tolerance. Their further investigation may well provide a useful field of future endeavour.

\section{References}

1 Hammermeister KE, Warbasse JR. The rate of change of left ventricular volume in man. II. Diastolic events in health and disease. Circulation 1974; 49: 739-47.

2 Reduto LA, Wickemeyer WJ, Young JB, et al. Left ventricular diastolic performance at rest and during exercise in patients with coronary artery disease. Assessment with first pass radionuclide angiography. Circulation 1981; 63: 1228-37.

3 Bonow RO, Bacharach SL, Green MV, et al. Impaired left ventricular diastolic filling in patients with coronary artery disease: assessment with radionuclide angiography. Circulation 1981; 64: 315-23.

4 Gibson DG, Brown DJ. Measurement of peak rates of left ventricular wall movement in man. Br Heart $\mathcal{Y} 1975$; 37: 677-83.

5 Dodge HT, Sandler H, Baxley WA, Hawley RR. Usefulness and limitations of radiographic methods for determining left ventricular volume. Am $\mathcal{F}$ Cardiol 1966; 18: $10-24$.

6 Gibson DG, Brown DJ. Continuous assessment of left ventricular shape in man. Br Heart $\mathcal{F}$ 1975; 37: 904-10.

7 Gibson DG, Prewitt TA, Brown DJ. Analysis of left ventricular wall movement during isovolumic relaxation and its relation to coronary artery disease. Br Heart $\mathcal{F} 1976$; 38: 1010-9.

8 Hui WKK, Lee PK, Chow JSF, Gibson DG. Analysis of regional left ventricular wall motion during diastole in mitral stenosis. Br Heart f, 1983; 50: 231-9.

9 Sanderson JE, Gibson DG, Brown DJ, Goodwin JF. Left ventricular filling in hypertrophic cardiomyopathy. An angiographic study. Br Heart $\mathcal{F}$ 1977; 39: 661-70.

10 Henderson Y, Scarbrough MM, Chillingworth FP. The volume curve of the ventricles of the mammalian heart, and the significance of this curve in respect to the mechanics of the heartbeat and the filling of the ventricles. Am F Physiol 1906; 16: 325-67.

11 Oldershaw PJ, Dawkins KD, Ward DE, Gibson DG. Diastolic mechanisms of impaired exercise tolerance in aortic valve disease. $\mathrm{Br}$ Heart $\mathcal{F}$ 1983; 49:568-73.

12 Wood P. An appreciation of mitral stenosis. Part II. Investigations and results. $\mathrm{Br} \mathrm{Med} \mathcal{F} 1954$; i: 1113-24.

13 Gibson DG, Traill TA, Brown DJ. Changes in left ventricular free wall thickness in patients with ischaemic heart disease. Br Heart $\mathcal{F}$ 1977; 39: 1312-8.

14 Mattheos M, Shapiro E, Oldershaw PJ, Sacchetti R, Gibson DG. Non-invasive assessment of changes in left ventricular relaxation by combined phono-, echo-, and mechanocardiography. Br Heart f 1982; 47: 253-60.

15 Dodek A, Kassebaum DG, Bristow JD. Pulmonary edema in coronary artery disease without cardiomegaly. Paradox of the stiff heart. $N$ Engl $\mathcal{F}$ Med 1972; 286: 1347-50.

16 Grossman W, McLaurin LP. Diastolic properties of the left ventricle. Ann Intern Med 1976; 84: 316-26.

17 Grossman W, Mann T. Evidence for impaired left ventricular relaxation during acute ischemia in man. Eur $\mathcal{F}$ Cardiol 1978; 7 (suppl): 239-49.

18 Shapiro E, Marier DL, St John Sutton MG, Gibson DG. Regional non-uniformity of wall dynamics in normal left ventricle. Br Heart $\mathcal{f}$ 1981; 45: 264-70.

19 Gibson DG, Greenbaum R, Marier DL, Brown DJ. Clinical significance of early diastolic changes in left ventricular wall thickness. Eur Heart $\mathcal{F}$ 1980; 1 (suppl A): $157-63$.

Requests for reprints to Dr D G Gibson, Cardiac Department, Brompton Hospital, Fulham Road, London SW3 6HP. 